\title{
1 Combined analysis of the time-resolved transcriptome and proteome of plant
}

\section{pathogen Xanthomonas oryzae pv. oryzae}

4 Seunghwan Kim ${ }^{1}$, Wooyoung Eric Jang ${ }^{2}$, Min-Sik Kim ${ }^{2,3}$, Jeong-Gu Kim ${ }^{1 \S}$, Lin-Woo Kang ${ }^{4 \S}$ 5

$6{ }^{1}$ Genomics Division, National Institute of Agricultural Sciences (NAS), Rural Development 7 Administration (RDA), Jeonju 54874, Republic of Korea; ${ }^{2}$ Department of Applied Chemistry, 8 Kyung Hee University, Yongin 17104, Republic of Korea; ${ }^{3}$ Department of New Biology, 9 DGIST, Daegu 42988, Republic of Korea; ${ }^{4}$ Department of Biological Sciences, Konkuk 10 University, 1 Hwayang dong, Gwangjin-gu, Seoul 05029, Republic of Korea

$13{ }^{\S}$ Corresponding authors:

14 Dr. Lin-Woo Kang

15 Department of Biological Sciences, Konkuk University, Seoul 05029, Republic of Korea; Tel.

Dr. Jeong-Gu Kim

19 Genomics Division, National Academy of Agricultural Science (NAAS), Rural Development 20 Administration (RDA), Jeonju 54874, Republic of Korea; Tel. 82-63-238-4566; E-mail: 21 jkim5aug@korea.kr

23 Dr. Min-Sik Kim, Department of New Biology, DGIST, Daegu 42988, Republic of Korea; E24 mail: $\underline{\text { mkim@dgist.ac.kr }}$ 
26 Running title: analysis of transcriptome and proteome in P-activated Xoo 


\section{Abstract}

29 Xanthomonas oryzae pv. oryzae (Xoo) is a plant pathogen responsible for causing bacterial 30 blight in rice. The immediate alterations in Xoo upon initial contact with rice are essential for 31 pathogenesis. We studied time-resolved genome-wide gene expression in pathogenicity32 activated Xoo cells at the transcriptome and proteome levels. The early response genes of Xoo 33 include genes related to cell motility, inorganic ion transport, and effectors. The alteration of 34 gene expression is initiated as early as few minutes after the initial interaction and changes with 35 time. The time-resolved comparison of the transcriptome and proteome shows the differences 36 between transcriptional and translational expression peaks in many genes, although the overall 37 expression pattern of mRNAs and proteins is conserved. The discrepancy suggests an important 38 role of translational regulation in Xoo at the early stages of pathogenesis. The gene expression analysis using time-resolved transcriptome and proteome provides unprecedented valuable information regarding Xoo pathogenesis.

42 Keywords: Xoo-rice interactions, proteome and transcriptome, time-resolved gene expression,

43 Xanthomonas oryzae pv. oryzae, pathogenicity, translational regulation 


\section{Introduction}

Rice (Oryza sativa L.) is the most widely consumed staple food, sustaining two-thirds of the world's population (Jackson, 2016). Bacterial blight of rice caused by Xanthomonas oryzae pv. oryzae (Xoo) is a devastating disease for which an effective pesticide has not been developed yet; it is known to cause severe yield losses of up to $50 \%$ in several rice-growing countries

50 (Oliva et al., 2019). The demand for rice is expected to increase by at least $25 \%$ by 2030, owing to the rapidly growing world population, environmental stress arising in response to climate change, and pathogen pressure (Li, Wang, \& Zeigler, 2014). Further, the Green Revolution has resulted in a shift in rice cultivation, from varied traditional landraces to limited high-yielding

54 varieties, via artificial selection. This has resulted in the co-evolution of crop pathogens including Xoo with the selected host races in the modern agricultural ecosystem (Quibod et al., 2020).

The pathogen-host system of Xoo and rice serves as an ideal agricultural model to study crop diseases in a field setting at the molecular level; this is facilitated by the early elucidation of the whole genome structure of both Xoo and rice (Jackson, 2016; Lee et al., 2005). Xoo typically invades rice leaves through the wounds or hydathodes and replicates in the xylem vessels to cause disease (Mew, Alvarez, Leach, \& Swings, 1993). Rice contains a two-tiered innate immune system, consisting of pathogen-associated molecular pattern- and effectortriggered immunity, which protects against Xoo and initiates the hypersensitivity response at

64 the infection site (Jones \& Dangl, 2006). In Xoo-rice interactions, Xoo injects effectors into rice cells to modulate the cellular activities of the host to promote pathogenesis (Tsuge, Furutani, \& Ikawa, 2014). The early interactions between Xoo and rice at the infection site determine the fate of infection, i.e., occurrence of disease or initiation of the immune response. 
understanding of alterations in the Xoo cells in response to the initial interactions with rice is limited.

71 Transcription and translation are tightly coupled in bacteria and can occur simultaneously in

72 the cytosol (Hershey, Sonenberg, \& Mathews, 2019). Although proteins are the final functional

73 products of genes, the quantity of specific mRNA molecules often represents the expression

74 level of a gene at a given time point with the well-established RNA sequencing (RNA-Seq)

75 technology. In comparison, high resolution mass spectrometry-based quantitative proteomics

76 is a more recent analytical technique, and still has a lower coverage of protein products,

77 requires greater sample quantity, and is more expensive (Schubert, Rost, Collins, Rosenberger,

78 \& Aebersold, 2017).

79 We had previously developed an in vitro pathogenicity assay to recapitulate Xoo-rice interactions at the site of infection by treating Xoo cells with the rice homogenate (RLX), and assessed the time-resolved changes in the transcriptome (S. Kim et al., 2016; S. H. Kim et al., 2011; S. Kim et al., 2013). The in vitro pathogenicity assay provides high signal to noise data with Xoo cells synchronized with respect to the timing of pathogenicity activation.

84 Transcriptome data from RNA-Seq experiments revealed that most virulence genes of Xoo were upregulated within an hour of the initial interaction with RLX, and these upregulated genes were related to bacterial motility, inorganic ion transport, hypersensitive response and pathogenicity (hrp), bacterial toxins and effectors of avirulence (avr), plant cell wall degradation, and extracellular polysaccharide synthesis and secretion (S. Kim et al., 2016). from the transcriptome to the proteome. The time-dependent expression of specific mRNAs 
bioRxiv preprint doi: https://doi.org/10.1101/2020.10.02.324541; this version posted January 30, 2021. The copyright holder for this preprint (which was not certified by peer review) is the author/funder. All rights reserved. No reuse allowed without permission.

93 Xoo.

94 


\section{Results}

\section{Time-resolved proteome data}

97 We coupled LC-MS/MS technology with an in vitro assay system to obtain the time-resolved proteome data for P-activated Xoo cells (Scheme 1). The in vitro assay system recapitulated the initial interaction between Xoo cells and damaged rice leaf tissues at the site of infection; this was achieved by adding fresh RLX — prepared by grinding the leaves of a Xoo-susceptible rice cultivar (Milyang 23) in liquid nitrogen - to a Xoo cell culture in the mid-exponential

102 phase. Samples for proteome analysis were collected from RLX-treated (P-activated) and untreated (control) Xoo cells at 0, 30, 60, 90, and 120 min after RLX treatment (Table S1). genome (KACC10331) corresponded to 4,382 proteins in the proteome, of which 2,589 proteins $(59 \%)$ were identified for at least one time point and 2,296 proteins $(52 \%)$ were

107 detected in both replicates (Figure S1A). Median sequence coverages for total identified 108 proteins were $24 \%$ and $23 \%$ for each of the independent duplicate experiments (Figure S1B). A total of 20,963 and 19,684 non-redundant peptides were identified in both replicates, with 47,025 and 41,428 peptide-spectrum matches, respectively (Figure S1C).

111 Protein abundance values obtained after quantile-normalization (Figure S2A) were used for 112 pairwise comparisons; the Pearson's correlation coefficients corresponding to the abundance 113 values showed close correlations (0.98-0.99), indicating comparable cellular concentration of 114 most proteins (Figure S2B). The smallest correlations were observed for the RLX-treated 115 samples at $30 \mathrm{~min}$, indicating greater changes in the proteome during the initial $30 \mathrm{~min}$; this 116 was consistent with the transcriptome data. We further performed a principal component 117 analysis to determine the relationships between the assessed samples. Figure S2C shows that 118 the P-activated sample at 0 min clustered closely with all control samples, whereas the P- 
119 activated samples at other time points were more spread out. The proteome of the P-activated

120 Xoo cells at 30 min was considerably different from that of P-activated Xoo at other time points;

121 this was consistent with the results of the pairwise multi-scatter plot (Figure S2B).

122 Time-supervised hierarchical clustering was performed to determine temporal and

123 synchronized changes in protein abundance. Two distinct synchronized patterns-one

124 decreasing and the other increasing in response to RLX treatment—were observed (Figure 1).

125 The two lists including proteins exhibiting the two patterns were used for STRING analysis,

126 which produced two interaction networks (Figure S3A-B). Several genes related to cell motility,

127 inorganic ion transport, and transcriptional regulators were immediately responsive to the

128 pathogenicity signal (Table S2); these have been described in detail in the discussion section.

\section{Up and down regulated proteins}

131 Quantitative proteome data obtained from P-activated and control Xoo cells at every $30 \mathrm{~min}$

132 allowed the visualization of the three-dimensional protein expression data in terms of the genes,

133 time intervals, and expression levels (Figure 2 and Table S3). The expressed protein level of

134 each gene from P-activated Xoo cells are divided by that of the same gene from control at each

135 time point to calculate the fold change of time-dependent protein expression level of the

136 specific gene. For all the open reading frames, approximately 93 (2.0\%), 213 (4.5\%), and 468

137 (9.9\%) proteins were upregulated by more than $200 \%$ (2-fold), $50 \%$, and $20 \%$ at $30 \mathrm{~min}$,

138 respectively, and approximately $7(0.1 \%), 93(2.0 \%)$, and $561(11.9 \%)$ proteins were

139 downregulated to less than $25 \%$ (2-fold), $50 \%$, and $80 \%$ at $30 \mathrm{~min}$, respectively (Table S4).

140 In case of the duration of expressed proteins in P-activated Xoo, only $8(0.2 \%), 32(0.7 \%)$,

141 and $75(1.6 \%)$ proteins were upregulated for entire $120 \mathrm{~min}$ by more than $200 \%$ (2-fold), $50 \%$,

142 and $20 \%$, respectively and $0(0 \%), 1(0.02 \%)$, and $87(1.8 \%)$ proteins were downregulated for 
143 the same 120 min to less than $25 \%$ (2-fold), $50 \%$, and $80 \%$, respectively (Table S5), indicating

144 that most proteins were temporarily up- or downregulated in the in vitro assay.

145 The proteins at $30 \mathrm{~min}$ presented the highest change at the expression level within $120 \mathrm{~min}$.

146 More than 90 proteins $(1.9 \%)$ were upregulated by more than 2-fold at $30 \mathrm{~min}$ in each dataset,

147 whereas 27 proteins were upregulated in both datasets from the duplicate experiments (Table

148 S6). Almost half of the 27 upregulated proteins were related to cell motility and ion uptake,

149 seven were related to chemotaxis and motility, and five were related to transporters and pumps.

150 At the same 30 min, more than 90 proteins were downregulated by more than $50 \%$, 17 proteins

151 were downregulated in both datasets (Table S7), including transcription-related proteins—such

152 as sigma-54 modulation protein and MetE/MetH family transcriptional regulator-and cell

153 division- and cell cycle-related proteins.

\section{Clusters of Orthologous Groups of proteins (COGs)}

156 To study the global gene expression pattern based on gene function, we superimposed time-

157 dependent protein expression levels as per the functional categories in the Clusters of

158 Orthologous Groups of proteins (COGs) database (Figure 2), which were grouped into three

159 classes (red, yellow, and blue) depending on the observed pattern. To facilitate the comparison

160 of time-dependent protein expression levels, the expression level at $0 \mathrm{~min}$ was set as the

161 reference level $(=1)$ for each gene, and the fold change in the protein expression level was

162 calculated at each time point, as in case of the RNA-Seq data analysis (S. Kim et al., 2016).

163 The most prominent changes in protein expression in Xoo cells were observed for proteins

164 associated with cell motility $(\mathrm{N})$ and inorganic ion transport and metabolism $(\mathrm{P})$, which were

165 placed in the red class. In category N, two major expression peaks, indicating more than 2-fold

166 up-regulation, were detected at 30 and $90 \mathrm{~min}$. In category $\mathrm{P}$, upregulated proteins peaked at 
167 around $30 \mathrm{~min}$. Proteins grouped in the yellow class exhibited moderate changes in their

168 expression level, and were divided into nine functional categories, including signal 169 transduction $(\mathrm{T})$, intracellular trafficking, secretion, and vesicular transport (U), energy 170 production and conversion $(\mathrm{C})$, transcription $(\mathrm{K})$, replication $(\mathrm{L})$, cell wall/membrane/envelop 171 biogenesis $(\mathrm{M})$, and protein turnover $(\mathrm{O})$. The blue class included proteins associated with other 172 nine categories, the expression levels of which exhibited little change when compared with 173 those at 0 min, albeit except for a few proteins.

174

\section{Comparison of mRNA and protein levels}

176 We compared mRNA and protein levels of each gene in the transcriptome and proteome 177 (Figure 3; thin dashed red lines of mRNAs and thick dashed and solid black lines of proteins).

178 Both transcriptome and proteome data were obtained for up to 120 min after RLX treatment.

179 The experimental procedure used to prepare protein samples for LS-MS/MS required at least $18030 \mathrm{~min}$, whereas that for mRNA samples required at least $5 \mathrm{~min}$; the time interval for the 181 proteome data was set to $30 \mathrm{~min}$, whereas that for transcriptome data was set to 5-30 min. Due 182 to the different sampling times, the transcriptome data included mRNA levels at additional time 183 points of $5,10,15$, and $45 \mathrm{~min}$, whereas the proteome data did not include protein levels 184 between 0 and $30 \mathrm{~min}$, in which case the protein levels (thick dashed black lines) were 185 extrapolated based on the protein levels at other time points using non-linear regression.

187 Flagella and chemotaxis-related genes

188 Flagella and chemotaxis-related genes encode more than 40 proteins, including structural 189 components and assembly factors of flagellar hook-basal body and filament and chemotaxis 190 proteins (Mukherjee \& Kearns, 2014). Two dominant hierarchical clusters, with distinct 
synchronized patterns of increasing and decreasing proteome concentration (indicated by red and blue, respectively), were identified (Figure 1). The red cluster of 359 proteins included 21 $(53 \%)$ of the 40 genes related to the flagellar assembly pathway, of which 20 genes were 194 included in the red cluster (Table S2).

195 We grouped the flagella and chemotaxis-related genes into three gene clusters, i.e., groups 196 I-A, I-B, and II, based on their positions in the genome (Figure 4A). Genes in cluster I-A and 197 I-B were associated with flagellar machinery, such as flagellar basal body hook and type III secretion system (T3SS), whereas cluster II included chemotaxis-related genes. clearly superimposed upregulated peaks at 30 min (Figure 3A). The transcriptome data revealed that most flagella and chemotaxis-related genes were regulated in a similar pattern, i.e., genes in all clusters of I-A, I-B, and II were downregulated at 5 min and upregulated at 30 $\min$. However, the proteome data presented a different expression pattern between clusters I-A and I-B and cluster II. Proteins in clusters I-A and I-B were upregulated at $30 \mathrm{~min}$, consistent with the transcriptome data. But proteins in cluster II were upregulated at $90 \mathrm{~min}$, exhibiting a delay of $1 \mathrm{~h}$ when compared with the transcriptome data (Figure S4).

207

\section{Inorganic ion transport and metabolism genes}

209 Iron uptake genes play a key role in pathogenicity during the early stages of host-pathogen 210 interactions (Garau et al., 2004). TonB-dependent receptors (TBDRs) are bacterial outer 211 membrane proteins that bind and transport ferric chelates of siderophores. Several annotated 212 TBDR genes have been identified in the Xoo KACC10331 genome, including IroN, FyuA, $213 F e c A, B t u B, F h u A$, CirA, and FepA (S. Kim et al., 2016). Of these, FecA (Xoo0901) and CirA 214 (Xoo3793) were upregulated and IroN genes (Xoo0394 and Xoo1784) were downregulated in 
215 both the transcriptome and proteome data. In the proteome data, FecA (Xoo0901) and CirA

216 (Xoo3793) upregulation peaked at $30 \mathrm{~min}$ (Figure 5A). IroN genes that were downregulated at

217 the transcript level were also downregulated at the protein level (Figure 5B). The expression

218 levels of other paralogs of FecA and CirA genes were comparable with those in the control in

219 the transcriptome as well as the proteome data (Figure S5), indicating that these genes could

220 respond to different pathogenic signals - which were missing in the in vitro system —or be

221 pseudogenes.

222 Phosphate uptake genes, i.e., OprO, PhoX, PstSCAB, and PhoU, were upregulated in the 223 transcriptome (up to 16 -fold) at 5-10 min. The proteome data of OprO, PhoX, PstSCAB, and 224 PhoU revealed upregulation up to 3-fold at $30 \mathrm{~min}$ (Figure S6).

227 In Xanthomonas oryzae pv. oryzicola, AvrBs2 suppresses host immunity and promotes 228 disease development (Ullah et al., 1998). In Xanthomonas campestris pv. vesicatoria, the role 229 of AvrBs3 is that of a transcription activator-like (TAL) effector that activates the expression 230 of plant immunity genes (Kay, Hahn, Marois, Wieduwild, \& Bonas, 2009). The secretion of effectors XoAvrBs2 (Xoo0168) — a Xoo ortholog of AvrBs2 — and XoAvrBs3 (Xoo2276)—a Xoo ortholog of AvrBs3 - through T3SS was confirmed upon interaction with RLX (S. H. Kim et al., 2011; S. Kim et al., 2013). In addition to the transcriptome and proteome, the expression 234 and secretion of effectors were assessed using dot blots of Xoo cells transformed with the TAP235 tagged effector genes (Figure 6).

236 The expression of XoAvrBs 2 transcript was upregulated from 5-30 min, while that of the 237 XoAvrBs2 protein was upregulated at $30 \mathrm{~min}$, as is evident from the transcriptome and 238 proteome data. In the dot blots, the levels of TAP-tagged XoAvrBs2 were upregulated by 4- 
239 fold at $5 \mathrm{~min}$ and the secretion of XoAvrBs2 was detected as early as $15 \mathrm{~min}$. The expression 240 of $X o A v r B s 3$ transcript was upregulated and peaked from 5-30 min, whereas that of XoAvrBs3

241 protein was maintained at almost the same-or slightly lower levels — at $60 \mathrm{~min}$ in the

242 transcriptome and proteome data. Dot blots revealed the secretion of XoAvrBs3 from 15 min. 


\section{Discussion}

Plant pathogens could exhibit complex responses to the initial interactions with the host under varying biotic and abiotic conditions at the site of infection. The varied immediate

247 response of the plant pathogen is important for successful infection. In this study, we analyzed 248 the immediate time-resolved response of Xoo cells from the initial interaction with rice in terms 249 of gene expression at both the mRNA and protein levels using an in vitro assay system, wherein 250 RLX mimicked the damaged rice leaf tissue (Scheme 1).

251 Of all the predicted open reading frames in Xoo, we quantified approximately $83 \%$ and $47 \%$ 252 of the mRNAs and proteins, respectively, in a time-dependent manner. A good correspondence 253 of overall expression pattern was observed between the mRNA and protein levels of genes. The 254 expression pattern was more synchronized for genes closely located in the genome (Figure 4 and S4); this could be attributed to the polycistronic gene structure in bacteria. With respect to the rapidity of pathogenic gene expression in response to the signals triggered upon interaction with the host, both transcriptional and translational machineries responded immediately to the interaction, at the earliest assessed time points of 5 and $30 \mathrm{~min}$ in the transcriptome and proteome data. The secretion of effectors was also found at $15 \mathrm{~min}$ in dot blots. Both mRNAs and proteins presented the greatest variation in their levels during the initial $30 \mathrm{~min}$.

The Xoo genes exhibiting the most rapid responses to the initial interaction with RLX, included genes associated with cell motility and inorganic ion uptake, and genes coding for effector molecules. All these three functional categories of genes are closely related to the early stages of pathogenesis. The genes associated with cell motility could be responsible for the migration and accumulation of the Xoo cells at the site of infection via the damaged xylem tissues or exposed hydathodes in rice. As inorganic ions, such as iron and phosphate ions,

267 function as essential cofactors in all living organisms, bacterial pathogens and host rice cells 
compete to obtain and secure the limited resources available. Effector molecules are more directly related to pathogenesis and are injected by the Xoo into the rice cells via pili-like T3SS; these molecules modulate the immune system of rice.

271 The earliest available time-point for comparing the gene expressions in terms of mRNA and 272 protein levels was $30 \mathrm{~min}$ after the RLX treatment. At $30 \mathrm{~min}, 290 \mathrm{mRNAs}$ were upregulated 273 by more than 2-fold in the transcriptome, compared with 93 proteins in the proteome (Table 274 S4), and the average fold change in the expression of the upregulated genes in the transcriptome 275 and proteome was similar, i.e., 3.3-fold and 3.4-fold, respectively (Table S6). The number of 276 upregulated transcripts is much higher than - even though we figure into our calculations the 277 higher coverage of quantified genes in transcriptome than in proteome - that of the proteins at $27830 \mathrm{~min}$, which indicates that not all the upregulated mRNAs are simultaneously translated to 279 proteins.

280 The difference in the gene expression in terms of the mRNA and protein levels at a given 281 time point suggests the existence of an additional translational regulation step - after the 282 transcriptional regulation - although transcription and translation can occur simultaneously in 283 bacteria. For some genes, the mRNA and protein expression peaks were observed at varying 284 time points. Bacterial pathogenesis appears to involve a fine-tuning mechanism after transcription, which could help adjust the expression of the early-responsive genes under varying biotic and abiotic environmental conditions.

287 Genes related to flagella and cell motility are clustered in the bacterial genome. In Xoo, three 288 clusters are observed, i.e., I-A, I-B, and II. On superimposing the mRNA and protein levels in 289 time, the expression of mRNAs and proteins peaked at 30 mins for genes in the clusters I-A 290 and I-B, the expression of mRNAs and proteins peaked at 30 and $90 \mathrm{~min}$, respectively, for 291 genes in cluster II. A translational regulation step might be involved that determines the time 
292 for the translation of specific mRNAs — coded by flagella and cell motility-related genes — into 293 proteins depending on the priority of each gene.

294 The hierarchy of the expression of flagella and cell motility-related genes has been 295 extensively studied in the transcriptome of E. coli, where sigma and anti-sigma factors are 296 known to be the key transcriptional regulators (Mukherjee \& Kearns, 2014). The overall 297 organization of flagella and chemotaxis-related genes is different between E. coli and Xoo 298 (Figure S7). In Xoo, the cluster of $f l i E-R$ genes (cluster I-B) is positioned just downstream to the $f \lg B-L$ genes (cluster I-A), whereas in $E$. coli, chemotaxis genes are present between the two. $f l h D C$ genes do not have any orthologs in Xoo, and a different transcriptional regulator, 301 i.e., the fle $Q$ gene, is present. Based on the nomenclature used in E. coli, several Xoo genes 302 classified as Class III genes are located at different positions and their expression regulation is 303 also different. In Xoo, ribosomes or other translation factors might recognize certain unknown priority signals in mRNA transcripts for the translational regulation.

The immediate upregulation of inorganic ion uptake genes, especially iron uptake genes, may aid Xoo cells to obtain the essential cofactor ions, when they are competition with the host 307 cells (Liu, Kong, Wu, \& Ling, 2020). The iron ion is essential for photosynthesis and respiration and needed by many redox enzymes for most organisms on Earth. Pathogens commonly use iron chelating molecules or siderophores to derive this scarce inorganic cofactor from the hosts.

310 The leakage of iron ions from damaged leaf tissues might serve as an important signal of 311 initiating infection and might provide an opportunity to secure essential iron ions for the Xoo 312 cells.

313 In comparison with the flagella and cell motility-related genes that are closely clustered in 314 the genome and exhibit coordinated expression levels, the ion uptake genes are dispersed across 315 the genome and are independently expressed (Figure S8). The separation of these genes 
316 facilitates independent regulation. Compared with the flagella and motility-related genes, the

317 inorganic ion uptake genes exhibited great variation in the mRNA levels but similar protein

318 expression levels (Figure 3).

319 Effectors are key molecules for pathogenicity that modulate the host immune responses after

320 infection. The protein expression and secretion of the Xoo effectors XoAvrBs2 and XoAvrBs3

321 were assessed using dot blots, which enabled the monitoring of effector proteins at the early

322 stages of the initial interaction, i.e., within 30 min of RLX treatment. The secretion of effectors

323 was observed from $15 \mathrm{~min}$ after application of the pathogenic stimulus. In the P-activated

324 proteome data, the expression of effector proteins was maintained at a similar level to that at 0

$325 \mathrm{~min}$; this may be attributed to the similar protein synthesis and secretion rates.

326 The effector genes are also dispersed across the genome, like the ion uptake genes (Figure

327 S9). Interestingly, transposase genes are located close to the effector genes; these may aid the

328 effector genes to transpose through the bacterial genome and plasmids. The expression of

329 XoAvrBs2 was upregulated 5 min after RLX treatment, and its secretion was detected in the

330 culture medium at $15 \mathrm{~min}$. Although the cellular protein level was upregulated by

331 approximately $20 \%$ in the proteome at $30 \mathrm{~min}$, the dot blot showed an upregulation of 4-fold

332 at $5 \mathrm{~min}$. The secreted XoAvrBs2 exhibited a 16-fold increase at $120 \mathrm{~min}$. In case of XoAvrBs3,

333 the cellular protein level decreased by approximately $20 \%$ in the proteome at 60 min, whereas

334 the dot blot revealed a 16-fold increase in XoAvrBs3 secretion at $120 \mathrm{~min}$. The experimental

335 methods for proteome and dot blots analysis are different (S. H. Kim et al., 2011; S. Kim et al.,

336 2013). For the dot blots analysis, TAP-tagged XoAvrBs 2 and $X o A v r B s 3$ genes were introduced

337 into Xoo cells via the plasmid having the endogenous promotor and expressed from the plasmid.

338 In the proteome analysis, XoAvrBs2 and XoAvrBs3 proteins were expressed from the 339 endogenous $X o A v r B s 2$ and $X o A v r B s 3$ genes in the Xoo genome. 
340 Gene expression involves sequential transcription and translation. In bacteria, with respect

341 to post-transcriptional and post-translational modification, mRNAs without a cap at 5 ' end and

342 a poly A tail at $3^{\prime}$ end have a short half-life — as short as few minutes — and proteins undergo

343 only limited post-translational modifications. The limited post-translational modifications in

344 bacterial proteins impose a pressure on a nascent protein from the ribosome to take a functional

345 form immediately. The present study revealed that genes related to cell motility and inorganic

346 ion uptake, and genes coding for effector molecules of Xoo are the first to respond to the initial

347 interactions with RLX, and play an essential role in the following processes (Figure 7): (1)

348 invasion of the Xoo cells into the rice leaf tissues, (2) securing the limited cofactors, and (3)

349 modulating the immune responses of the host to favor pathogenesis. This combined analysis of

350 the time-resolved transcriptome and proteome of Xoo during the initial interaction with rice 351 tissues provides valuable insights into the pathogenic mechanism of Xoo. 


\section{Materials and Methods}

\section{Bacterial strain and culture conditions}

Xanthomonas oryzae pv. oryzae (Xoo) strain KACC10331, consisting of 4,941,439 nucleotides and 4,733 open reading frames, without any apparent autonomous plasmids, was

357 obtained from the Korean Agricultural Collection Center (KACC) (Lee et al., 2005). The 358 bacteria were cultured in nutrient broth (Difco, Detroit, MI, USA) or Yeast Glucose Cm Agar 359 (YGC) (1\% yeast extract, $2 \% \mathrm{D}-(+)$-glucose, $2 \% \mathrm{CaCO}_{3}$, and $1.5 \%$ agar) at $28^{\circ} \mathrm{C}$.

\section{Construction of expression vector and transformation of Xoo}

362 The effector genes XoAvrBs2 (Xoo0168) and XoAvrBs3-including the promoter region 363 (from -149 and -750 bp to the start codon of the respective gene) — were amplified by PCR and 364 ligated into the pGEM®-T Easy Vector (Promega). The cloned sequences were verified and then digested with $K p n I$ and $S a c I$, and the products were ligated into the pHM1-XTAP-T gap vector. The recombinant vectors were purified and introduced into Xoo strain KACC10331 by

367 electroporation, using Gene Pulser II (Bio-Rad, Hercules, CA) with a $0.2 \mathrm{~cm}$-gap cuvette at 2.5 $368 \mathrm{kV} \mathrm{cm}^{-1}$. Xoo cells were then diluted immediately with $1 \mathrm{~mL}$ Super Optimal Broth (SOC) medium and incubated at $28^{\circ} \mathrm{C}$ with agitation for $2 \mathrm{~h}$. Cells were then recovered from the culture medium and plated on nutrient broth agar plates containing $50 \mu \mathrm{L} \mathrm{mL}^{-1}$ spectinomycin and incubated at $28^{\circ} \mathrm{C}$ for $4 \mathrm{~d}$.

The transformants were cultured in $100 \mathrm{~mL}$ of nutrient broth up to the mid-exponential 373 phase $\left(\mathrm{A}_{600}=0.5\right)$. Cells were harvested by centrifuging $1 \mathrm{~mL}$ of the cell culture at $12,000 \mathrm{rpm}$ and $4{ }^{\circ} \mathrm{C}$ for $5 \mathrm{~min}$. Harvested cells were washed once with phosphate-buffered saline (PBS) at using $2 \mathrm{M}$ urea in PBS in 96-well plates and then transferred to a polyvinylidene difluoride 
377 membrane (PVDF; $0.2 \mu \mathrm{m}$, Bio-Rad) using a 96-well vacuum dot-blotter (Bio-Rad). The 378 membrane was then washed thrice with PBS, blocked with 5\% skim milk for 30 min, and 379 subjected to a one-step immuno-affinity reaction using the rabbit peroxidase-anti-peroxidase 380 soluble complex antibody (Sigma-Aldrich, St. Louis, USA). The membrane was developed, 381 and bound antibodies were detected by chemiluminescence.

\section{Treatment of rice leaf extract for proteome analysis}

Oryza sativa L. cv. Milyang 23, a Xoo-susceptible rice cultivar, was used for performing proteome analysis. Rice plants were grown in a paddy field at Jeonju in South Korea $\left(35^{\circ} 49^{\prime} 52.0^{\prime \prime} \mathrm{N} 127^{\circ} 03^{\prime} 55.6^{\prime \prime} \mathrm{E}\right)$ until panicle initiation (approximately 8 to 9 weeks). Forty clumped rice leaves were harvested and homogenized with liquid nitrogen using a mortar and pestle. One-gram aliquots of the resulting homogenate (RLX) were transferred to Eppendorf tubes and stored at $-80^{\circ} \mathrm{C}$. Xoo was cultured $(100 \mathrm{~mL})$ in nutrient broth up to the midexponential phase $\left(\mathrm{A}_{600}=0.5\right)$ in a shaking incubator at $28^{\circ} \mathrm{C}$ and $200 \mathrm{rpm}$, and RLX $(2 \mathrm{~g})$ was then added to the culture medium. The culture $(100 \mathrm{~mL})$ was subjected to sequential filtration through a gauze, $40-\mu \mathrm{m}$ nylon cell strainer (FALON, New York, USA), and 5- $\mu \mathrm{m}$ syringe filter (Sartorius, Germany) to remove $\operatorname{RLX}(0,30,60,90$, and 120 min after RLX addition). The filtered culture $(100 \mathrm{~mL})$ was centrifuged at $10,000 \times g$ and $4^{\circ} \mathrm{C}$ for $10 \mathrm{~min}$. Duplicate samples were obtained for each time point from two independent experiments.

\section{Sample preparation for proteome analysis}

398 The harvested samples were lysed in a lysis buffer (9 M urea prepared in $20 \mathrm{mM}$ HEPES (pH inhibitor (PhosSTOP, Sigma-Aldrich)) and sonicated on ice. The exact amount of proteins in 
401 each sample was determined using the bicinchoninic acid assay. Protein integrity was confirmed by SDS-PAGE and $200 \mu \mathrm{g}$ of protein from each sample was used for analysis. The

403 disulfide bonds were reduced by treatment with $10 \mathrm{mM}$ dithiothreitol for $1 \mathrm{~h}$, and incubation 404 with $30 \mathrm{mM}$ iodoacetamide (30 min in the dark) was performed to alkylate free sulfhydryl 405 functional groups. Samples were diluted with triethylammonium bicarbonate buffer (pH 8.0) 406 in a manner such that the final urea concentration was 1.5 M. Proteins were digested using MS 407 grade trypsin (Thermo Fisher Scientific) at a protein to enzyme ratio of $50: 1$ for $12 \mathrm{~h}$ at $37^{\circ} \mathrm{C}$. 408 The reaction was quenched by lowering the sample $\mathrm{pH}(<3)$ using trifluoroacetic acid. The 409 obtained peptides were desalted using a C18 spin column (Harvard) to remove salts and other 410 contaminants, and the purified peptides were dried. Then, they were isotopically labeled using 411 the 10-plex tandem mass tag (TMT, Thermo Fisher Scientific), as per the manufacturer's 412 protocol. The reaction was allowed to continue for $2 \mathrm{~h}$ at room temperature and TMT-labeled 413 samples were subsequently dried in a SpeedVac concentrator. Chemical labeling with TMT 414 was confirmed by liquid chromatography-tandem mass spectrometry (LC-MS/MS), and the 415 samples were pooled and fractionated using a basic reverse phase liquid chromatography 416 (RPLC) system. The pooled TMT-labeled peptide mixture was resuspended in $10 \mathrm{mM}$ 417 ammonium formate and fractionated into 12 fractions using a $\mathrm{C} 18$ column $\left(\mathrm{C}_{18}, 5 \mu \mathrm{m}\right.$ pore size, $4184.6 \mathrm{~mm} \times 250 \mathrm{~mm}$, XBridge, Waters). The fractionated peptides were dried and stored at $-80^{\circ} \mathrm{C}$ 419 until LC-MS/MS analysis.

420

$421 \quad$ LC-MS/MS and database search

422 Each fractionated peptide sample was analyzed using an Orbitrap Fusion ${ }^{\mathrm{TM}}$ Lumos $^{\mathrm{TM}}$ Tribrid $^{\mathrm{TM}}$ 423 Mass Spectrometer coupled with the Easy-nLC 1200 nano-flow liquid chromatography system 424 (Thermo Fisher Scientific). The dried peptides were reconstituted using $0.1 \%$ formic acid and 
425 loaded on a C18 trap column. Peptides were resolved using a linear gradient solvent B (0.1\%

426 formic acid in 95\% acetonitrile) and analyzed by high resolution mass spectrometry in the data-

427 dependent acquisition mode. MS1 and MS2 were acquired for the precursor and the peptide

428 fragmentation ions, respectively. MS1 scans were measured at a resolution of 120,000 and

429 an $m / z$ of 200 . MS2 scans were acquired following the fragmentation of precursor ions by high-

430 energy collisional dissociation (HCD) and were detected at a mass resolution of 50,000 and

431 an $m / z$ of 200 . Dynamic exclusion was used to reduce redundant fragmentation of the same

432 ions. The obtained mass spectrometry data were analyzed using the MaxQuant software

433 (Tyanova, Temu, \& Cox, 2016). Raw MS data were searched against the Xoo proteome in

434 UniProt database. Carbamidomethylation of cysteine and 10-plex TMT modification of lysine

435 and N-terminals were set as static modifications, whereas oxidation of methionine was set as a

436 variable modification. False discovery rates at the levels of protein and peptide-spectrum

437 matches were set at 0.01 . The raw MS data and MaxQuant search results have been submitted 438 to ProteomeXchange (project accession: PXD020135, reviewer access

439 with username: reviewer34070@ebi.ac.uk and password: mzn76I1O).

\section{$441 \quad$ Proteomic data analysis}

442 The contaminant and reverse identified proteins were removed from the MaxQuant data.

443 Proteins identified in both replicates were pooled for quantile normalization. The normalized

444 values for the replicates were subjected to supervised hierarchical clustering and principal 445 component analysis, using Perseus (Tyanova, Temu, Sinitcyn, et al., 2016), and the results were 446 depicted in the form of a multi-scatter plot.

\section{STRING map analysis}


449 The list of proteins in the selected patterns from the hierarchical clusters was uploaded on the

450 STRING database (https://string-db.org/) to analyze the protein interaction maps. The clusters

451 of proteins in the blue and red classes, which show the similar time-resolved expression

452 patterns, were analyzed, and the list including the gene names with the selected organism was

453 inputted in the multiple proteins search setting. The number of nodes and edges were

454 automatically calculated based on Xoo genes with a PPI enrichment p-value of 20.9E-10.

455 Figures were downloaded in the PNG file format for visualization.

456

457 RNA-Seq and data analysis

458 In addition to the previously obtained RNA-Seq data for P-activated Xoo cells, RNA-Seq data 459 at 90 and 120 min were obtained to correspond with the proteome data at these time points.

460 RNA-Seq and data analysis were performed as previously described (S. Kim et al., 2016).

461 Briefly, total RNA from samples was used to generate sequencing libraries, from which

462 ribosomal RNA was removed using MICROBExpress Bacterial mRNA Enrichment Kit

463 (Ambion, Austin, TX, USA), and enriched mRNA was prepared using Illumina TruSeq RNA

464 Sample Preparation Kit (Illumina, San Diego, CA, USA). The RNA obtained after 465 fragmentation was used to generate cDNA fragments, which were sequenced using Illumina 466 Genome Analyzer IIx and mapped to the reference genome sequence

467 (http://www.ncbi.nlm.nih.gov/nuccore/58579623?report=fasta) using CLC Genomics

468 Workbench 4.0 (CLC bio, Aarhus, Denmark). Relative transcript abundance was calculated 469 based on the number of reads per kilobase per million mapped sequence reads (RPKM).

470

471 Analysis of time-resolved continuous mRNA and protein expression

472 The RPKM values of the mRNAs in the transcriptome and TMT intensities of the proteins 
473 in the proteome corresponded to the observed expression level of each gene at a given time

474 point. To facilitate the comparison of gene expression levels, the observed expression level at

475 each time point was converted to fold change in gene expression, by dividing the expression

476 level at a given time point by the initial expression level $(0 \mathrm{~min})$ of the same gene. The fold

477 change in the time-resolved expression levels of a given gene during the two hours following

478 the RLX treatment was fitted to a curve and analyzed using non-linear regression by GraphPad

479 Prism (version 3.02 for Windows, GraphPad Software, San Diego California USA,

480 www.graphpad.com), and the continuous time-dependent changes in the mRNA and protein

481 expression levels were determined using the fitted curve.

482

483 Analysis of the correspondence between mRNA and protein expression levels

484 For the accurate comparison of the mRNA and protein expression levels in P-activated Xoo 485 cells, the expression levels were corrected by comparing with those in the control cells at each 486 time point. The fold change in mRNA and protein expression in P-activated Xoo cells at a given 487 time point was divided by that of the control cells at the same time point. For analyzing the 488 correspondence between the mRNA and protein levels of each gene in the transcriptome and 489 proteome, the resulting control-corrected fold change values of mRNAs were compared with 490 those of the proteins for a given time point as the gene expression level of mRNAs and proteins. 


\section{Declarations}

\section{Competing interests}

495 Seunghwan Kim, Wooyoung Eric Jang, Min-Sik Kim, Jeong-Gu Kim, and Lin-Woo Kang

496 declare that they have no conflict of interest. The authors declare no competing financial 497 interests. This article does not contain any studies with human or animal subjects performed 498 by the any of the authors.

499

\section{Available data and material}

501 The raw MS data and MaxQuant search results are available at ProteomeXchange (Project 502 accession: PXD020135, Reviewer access with username: reviewer34070@ebi.ac.uk 503 and password: mzn76I1O)

504

\section{Funding}

506 This work was undertaken in association with the Cooperative Research Program for

507 Agriculture Science \& Technology Development (Project No. PJ01327002020), Rural 508 Development Administration, Republic of Korea.

509

\section{Author Contributions}

511 Investigation, Seunghwan Kim, Wooyoung Eric Jang, Min-Sik Kim, Jeong-Gu Kim, and

512 Lin-Woo Kang; writing, Seunghwan Kim, Wooyoung Eric Jang, Min-Sik Kim, Jeong-Gu Kim, 513 and Lin-Woo Kang; methodology, Seunghwan Kim, Wooyoung Eric Jang, Min-Sik Kim, 514 Jeong-Gu Kim, and Lin-Woo Kang; funding acquisition, Jeong-Gu Kim, and Lin-Woo Kang; 515 supervision, Min-Sik Kim, Jeong-Gu Kim, and Lin-Woo Kang. All authors have read and 
516 agreed to the published version of the manuscript.

\section{Acknowledgments}

519 This study was supported by the Cooperative Research Program for Agriculture Science \&

520 Technology Development (Project No. PJ01327002020), Rural Development Administration

521 and by the 2021 RDA Fellowship Program of National Institute of Agricultural Sciences,

522 Rural Development Administration, Republic of Korea.

523

524

\section{Abbreviations}

526 Xoo, Xanthomonas oryzae pv. oryzae; RLX, rice leaf homogenate; KACC, Korean

527 Agricultural Collection Center; P-activated, Pathogenicity-activated; LC-MS/MS, liquid 528 chromatography-tandem mass spectrometry; COG, Clusters of Orthologous Groups; RPKM, 529 Reads per kilobase per million mapped reads

530

531 


\section{References}

534 Garau, G., Garcia-Saez, I., Bebrone, C., Anne, C., Mercuri, P., Galleni, M., ... Dideberg, O. (2004). Update of the standard numbering scheme for class B beta-lactamases. Antimicrob Agents Chemother, 48(7), 2347-2349. doi: 10.1128/AAC.48.7.23472349.2004

538

539

540

541

542 Cold Spring Harb Perspect Biol, 11(9). doi: 10.1101/cshperspect.a032607

Jackson, S. A. (2016). Rice: The First Crop Genome. Rice (N Y), 9(1), 14. doi: 10.1186/s12284016-0087-4

Jones, J. D., \& Dangl, J. L. (2006). The plant immune system. Nature, 444(7117), 323-329. doi: $10.1038 /$ nature 05286

Kay, S., Hahn, S., Marois, E., Wieduwild, R., \& Bonas, U. (2009). Detailed analysis of the DNA recognition motifs of the Xanthomonas type III effectors AvrBs3 and AvrBs3Deltarep16. Plant J, 59(6), 859-871. doi: 10.1111/j.1365-313X.2009.03922.x

Kim, S., Cho, Y. J., Song, E. S., Lee, S. H., Kim, J. G., \& Kang, L. W. (2016). Time-resolved pathogenic gene expression analysis of the plant pathogen Xanthomonas oryzae pv. oryzae. BMC Genomics, 17, 345. doi: 10.1186/s12864-016-2657-7

Kim, S. H., Lee, S. E., Hong, M. K., Song, N. H., Yoon, B., Viet, P., . . Kang, L. W. (2011). Homologous expression and quantitative analysis of T3SS-dependent secretion of TAPtagged XoAvrBs2 in Xanthomonas oryzae pv. oryzae induced by rice leaf extract. $J$ Microbiol Biotechnol, 21(7), 679-685.

Kim, S., Nguyen, T. D., Lee, J., Hong, M. K., Pham, T. V., Ahn, Y. J., . . Kang, L. W. (2013). Homologous expression and T3SS-dependent secretion of TAP-tagged Xo2276 in Xanthomonas oryzae pv. oryzae induced by rice leaf extract and its direct in vitro recognition of putative target DNA sequence. J Microbiol Biotechnol, 23(1), 22-28.

Lee, B. M., Park, Y. J., Park, D. S., Kang, H. W., Kim, J. G., Song, E. S., . . Go, S. J. (2005). The genome sequence of Xanthomonas oryzae pathovar oryzae KACC10331, the bacterial blight pathogen of rice. Nucleic Acids Res, 33(2), 577-586. doi: 10.1093/nar/gki206

Li, J. Y., Wang, J., \& Zeigler, R. S. (2014). The 3,000 rice genomes project: new opportunities and challenges for future rice research. Gigascience, 3, 8. doi: 10.1186/2047-217X-3-8 
Liu, Y., Kong, D., Wu, H. L., \& Ling, H. Q. (2020). Iron in Plant-pathogen Interactions. J Exp Bot. doi: 10.1093/jxb/eraa516

Mew, T. W., Alvarez, A. M., Leach, J. E., \& Swings, J. (1993). Focus on Bacterial-Blight of Rice. Plant Disease, 77(1), 5-12. doi: Doi 10.1094/Pd-77-0005

Mukherjee, S., \& Kearns, D. B. (2014). The structure and regulation of flagella in Bacillus subtilis. Annu Rev Genet, 48, 319-340. doi: 10.1146/annurev-genet-120213-092406

Oliva, R., Ji, C., Atienza-Grande, G., Huguet-Tapia, J. C., Perez-Quintero, A., Li, T., . . Yang, B. (2019). Broad-spectrum resistance to bacterial blight in rice using genome editing. Nat Biotechnol, 37(11), 1344-1350. doi: 10.1038/s41587-019-0267-z

Quibod, I. L., Atieza-Grande, G., Oreiro, E. G., Palmos, D., Nguyen, M. H., Coronejo, S. T., .. . Oliva, R. (2020). The Green Revolution shaped the population structure of the rice pathogen Xanthomonas oryzae pv. oryzae. ISME J, 14(2), 492-505. doi: 10.1038/s41396-019-0545-2

Schubert, O. T., Rost, H. L., Collins, B. C., Rosenberger, G., \& Aebersold, R. (2017). Quantitative proteomics: challenges and opportunities in basic and applied research. Nat Protoc, 12(7), 1289-1294. doi: 10.1038/nprot.2017.040

Tsuge, S., Furutani, A., \& Ikawa, Y. (2014). Regulatory network of hrp gene expression in Xanthomonas oryzae pv. oryzae. Journal of General Plant Pathology, 80(4), 303-313. doi: 10.1007/s10327-014-0525-3

Tyanova, S., Temu, T., \& Cox, J. (2016). The MaxQuant computational platform for mass 585 spectrometry-based shotgun proteomics. Nat Protoc, 11(12), 2301-2319. doi: 10.1038/nprot.2016.136

Tyanova, S., Temu, T., Sinitcyn, P., Carlson, A., Hein, M. Y., Geiger, T., . . Cox, J. (2016). The 588 Perseus computational platform for comprehensive analysis of (prote)omics data. Nat Methods, 13(9), 731-740. doi: 10.1038/nmeth.3901

Ullah, J. H., Walsh, T. R., Taylor, I. A., Emery, D. C., Verma, C. S., Gamblin, S. J., \& Spencer, J. (1998). The crystal structure of the L1 metallo-beta-lactamase from 593 594 


\section{Scheme legend}

597

598 Scheme 1. Schematic representation of the in vitro assay system and combined analysis of

599 the time-resolved transcriptome and proteome using RNA-Seq and LC-MS/MS

600

601 
Figure legends

603

604

Figure 1. Time-supervised hierarchical clustering of duplicate pathogenicity-activated

605

proteome datasets. The heatmap (left) of genes clustered based on the similarities in the

606 protein expression pattern over time is presented for duplicate samples of pathogenicityactivated and control Xoo cells. Low to high expression is indicated by a change in color from green to red. Selected cluster profile patterns (right) are presented for the downregulated (blue box) and upregulated (red box) genes in the P-activated Xoo cells.

610

611 Figure 2. Time-resolved protein expression patterns associated with the COG categories.

612 Two functional categories of genes (COG) associated with the greatest changes in the protein 613 level are indicated by red boxes, nine functional COGs associated with moderate changes are 614 indicated by yellow boxes, and other nine functional COGs associated with minor changes in 615 the protein level are indicated by blue boxes. The inset for each functional COG indicates the 616 control (untreated cells). The Y-axis represents the fold change in the protein expression level 617 in comparison with that at $0 \mathrm{~min}$, and the maximum value on the Y-axis was set as 3 . The $\mathrm{X}$ 618 axis indicates the time from 0 to $120 \mathrm{~min}$. Each line indicates the protein expression level of a 619 gene. The protein expression level is represented as grey (from 0 to $30 \mathrm{~min}$ ) and black (from 62030 to $120 \mathrm{~min}$ ) lines. Note that in contrast to the transcript data, the proteome data was not 621 available between 0 and 30 min (grey part).

623 Figure 3. Time-resolved mRNA and protein levels of genes associated with cell motility 624 and inorganic ion transport. (A) Time-resolved expression of cell motility-related genes. The 625 mRNA expression levels from RNA-Seq are indicated by dashed red lines. The protein 
626 expression levels from LC-MS/MS are indicated by black dashed (from 0 to 30 min) and black

627 solid (from 30 to $120 \mathrm{~min}$ ) lines. (B) Time-resolved expression of inorganic ion transport-

628 related genes. The mRNA expression levels from RNA-Seq and protein expression levels from

629 LC-MS/MS are indicated as in (A). The Y-axis represents the fold change.

630

631 Figure 4. Gene clusters and time-resolved expression patterns of cell motility-related

632 genes. (A) Gene clusters of flagellar biosynthesis-related genes (groups I-A and I-B) and

633 chemotaxis genes (group II). (B) Time-resolved mRNA and protein expression levels of genes

634 in groups I-A, I-B, and II. The down- and upregulation peaks are shown in green and red,

635 respectively. The yellow and grey ovals indicate unaltered and undetected expression levels.

636 The thin-bordered oval (at 5, 10, 15, and $45 \mathrm{~min}$ ) for the proteome data is to ensure consistency

637 with the pictorial format of RNA-Seq data. The dotted arrows and ovals indicate genes that are

638 not directly related to flagellar biosynthesis and chemotaxis. Time is expressed in min.

639

640 Figure 5. Time-resolved mRNA and protein levels of iron transport-related genes. (A)

641 Time-resolved mRNA (red) and protein (black) expression levels of FecA and CirA genes. (B)

642 Time-resolved mRNA (red) and protein (black) expression level of IroN genes. The Y-axis

643 represents $\log _{2}$ (fold-change).

644

645 Figure 6. Time-resolved mRNA and protein expression and secretion of effector proteins.

646 The mRNA (red) and protein (black) expression levels of XoAvrBs2 (A) and XoAvrBs3 (B)

647 genes in the transcriptome and proteome data (above) and the cytosolic and secreted XoAvrBs2

648 (A) and XoAvrBs3 (B) proteins in the dot blot data (below). NC, negative control; PC, positive 649 control 
651 Figure 7. Schematic representation of genome-wide pathogenic gene expression and

652 effector secretion via in vitro assay system. Early response Xoo genes from the initial

653 interaction with RLX include genes related to cell motility, iron uptake, and effector, of which

654 expression are upregulated as early as $5 \mathrm{~min}$ in mRNAs and $30 \mathrm{~min}$ in proteins and effector

655 secretion is found since $15 \mathrm{~min}$.

656 


\section{Scheme 1}

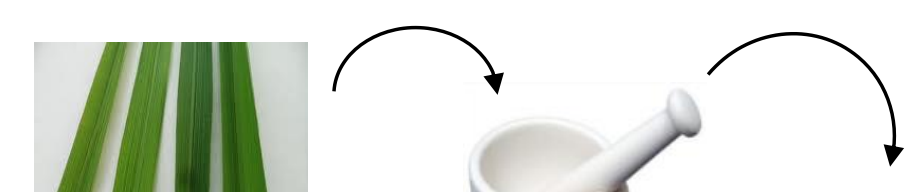

Rice leaf homogenate

Xoo-susceptible rice cultivar of Milyang23
Homogenization

in liquid $\mathrm{N}_{2}$
(RLX)

Initiation of interactions between RLX and Xoo

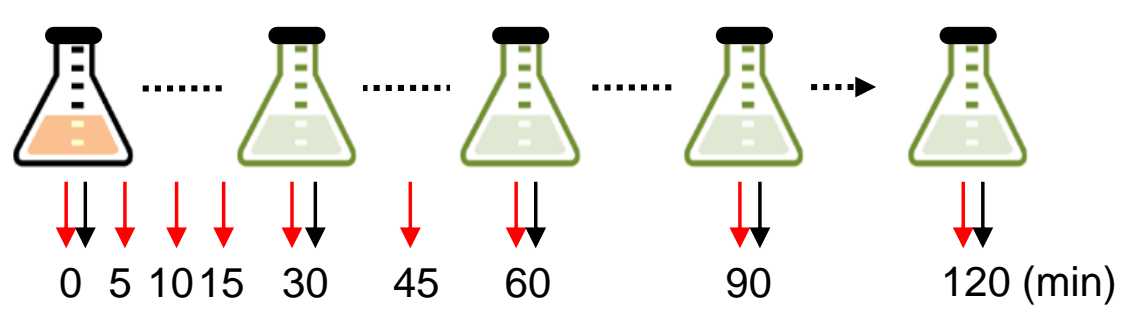

Time-resolved sampling of RNAs and proteins

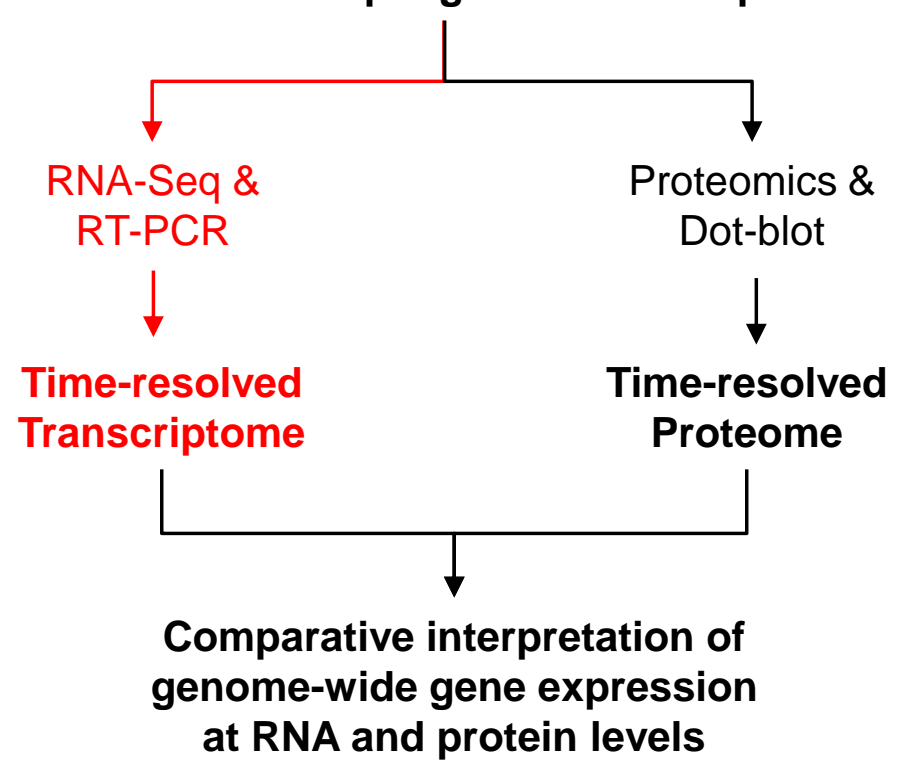




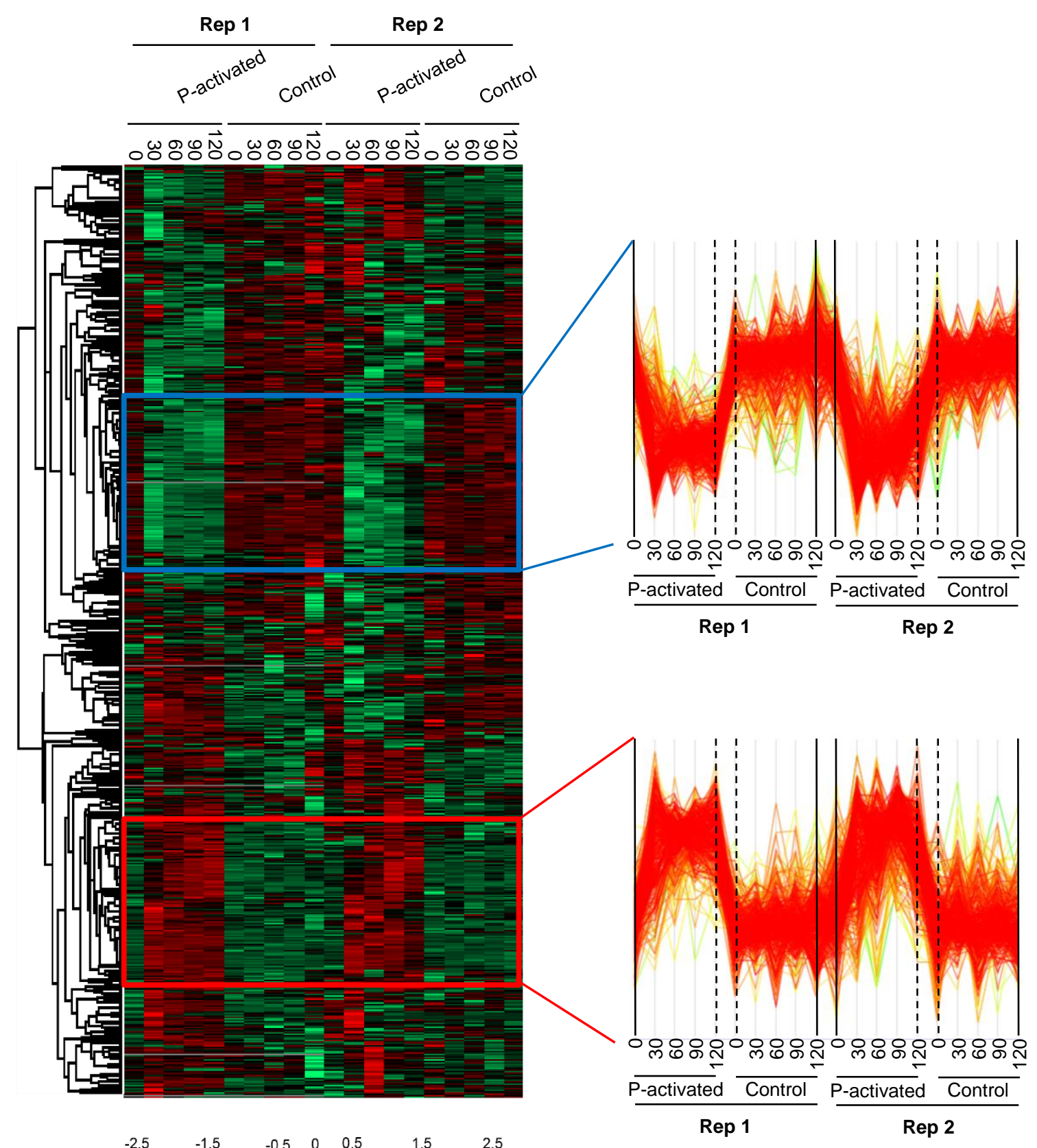




\section{Figure 2}
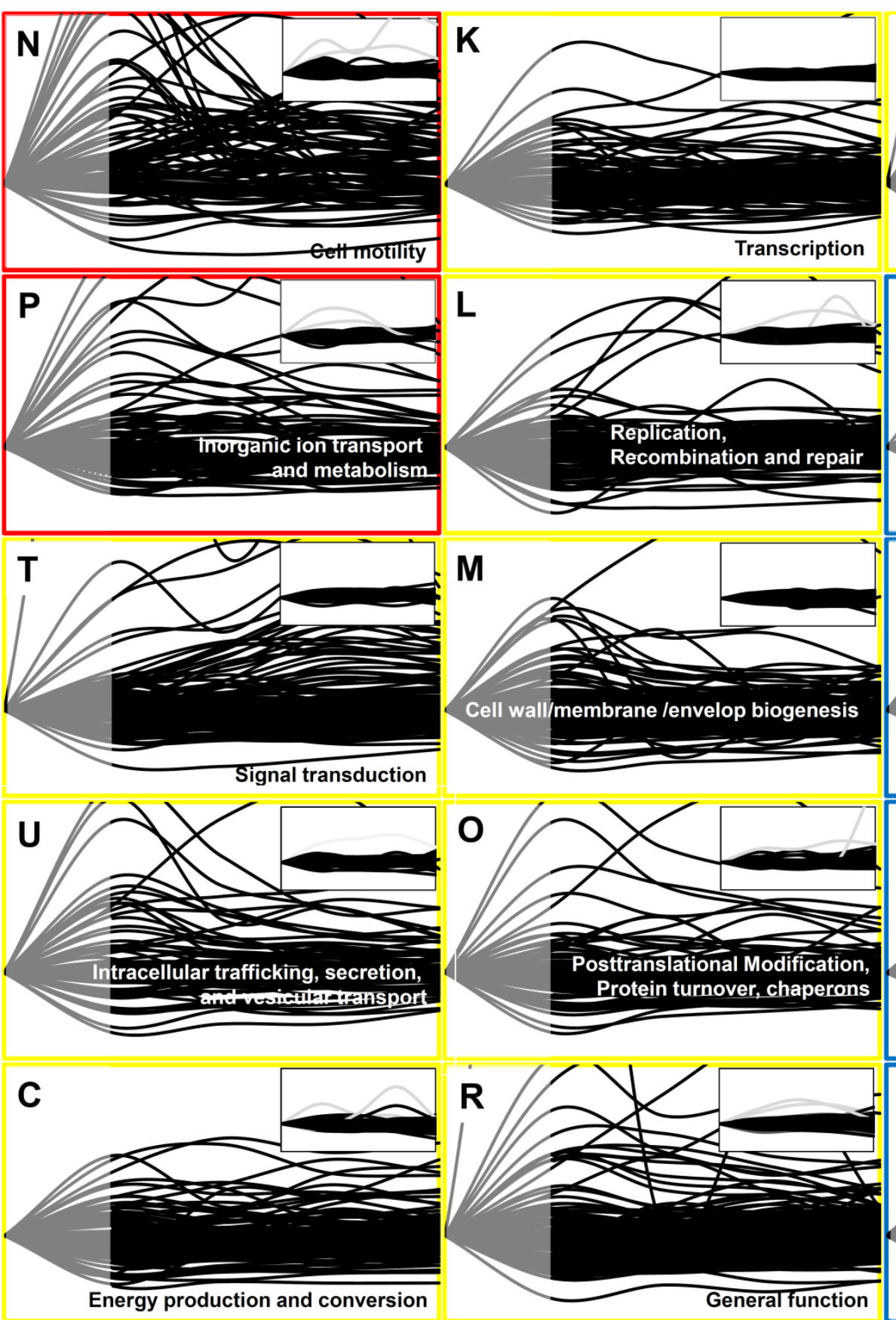
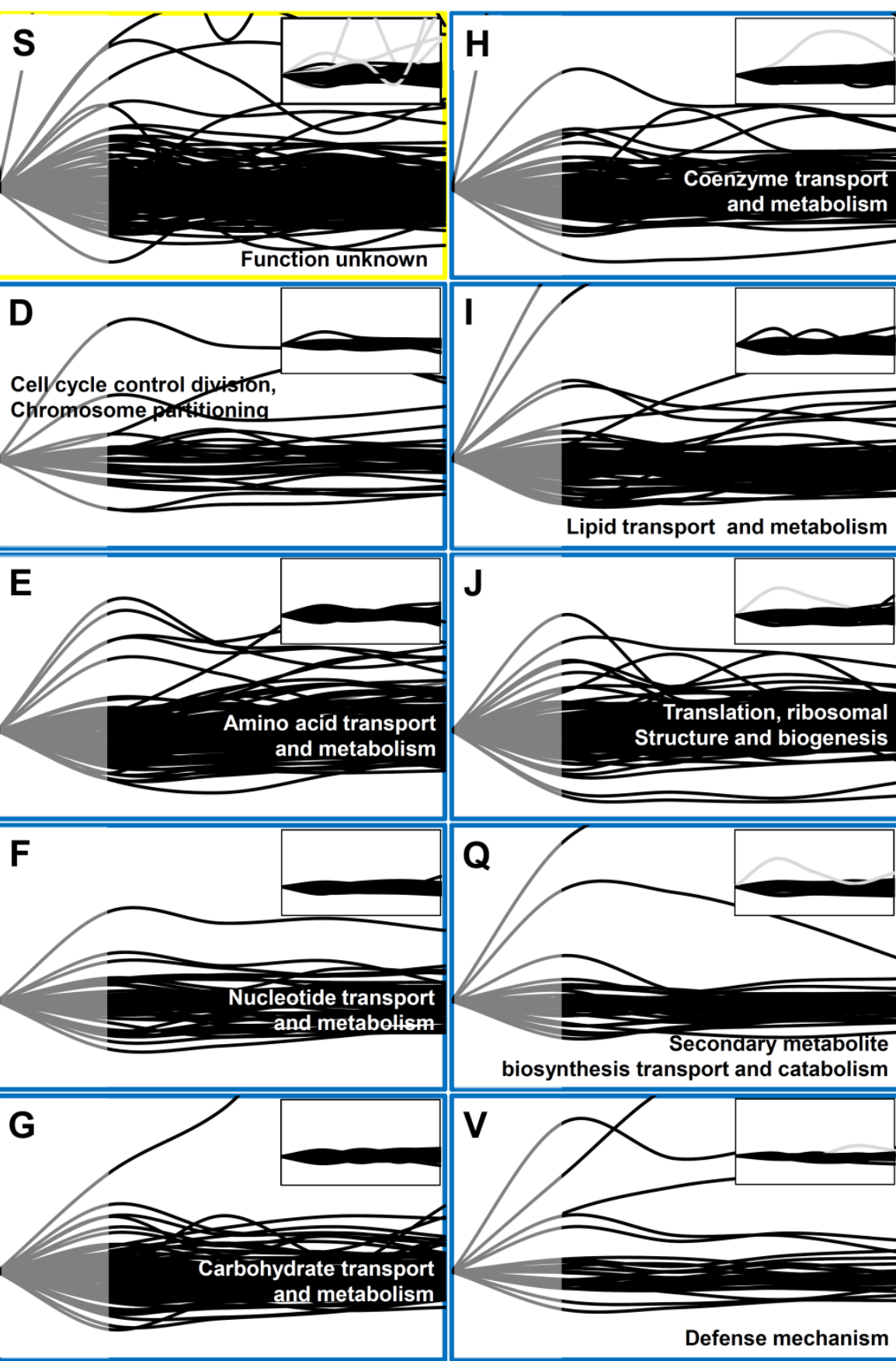
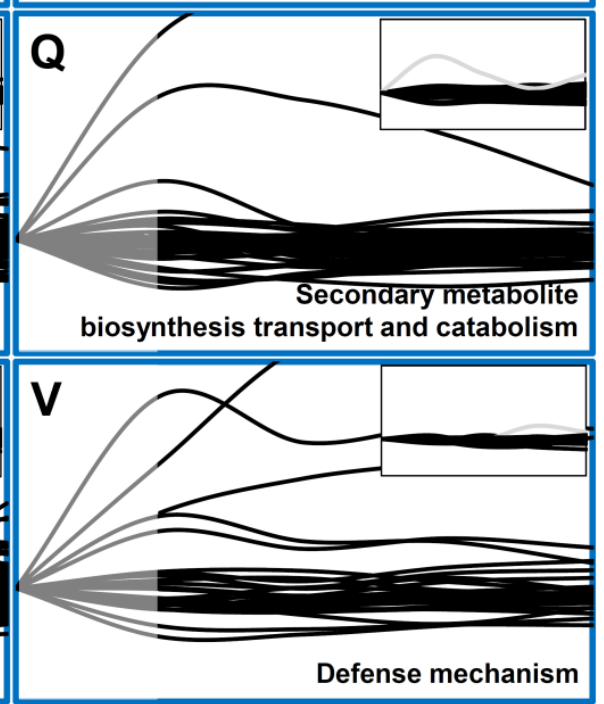
A

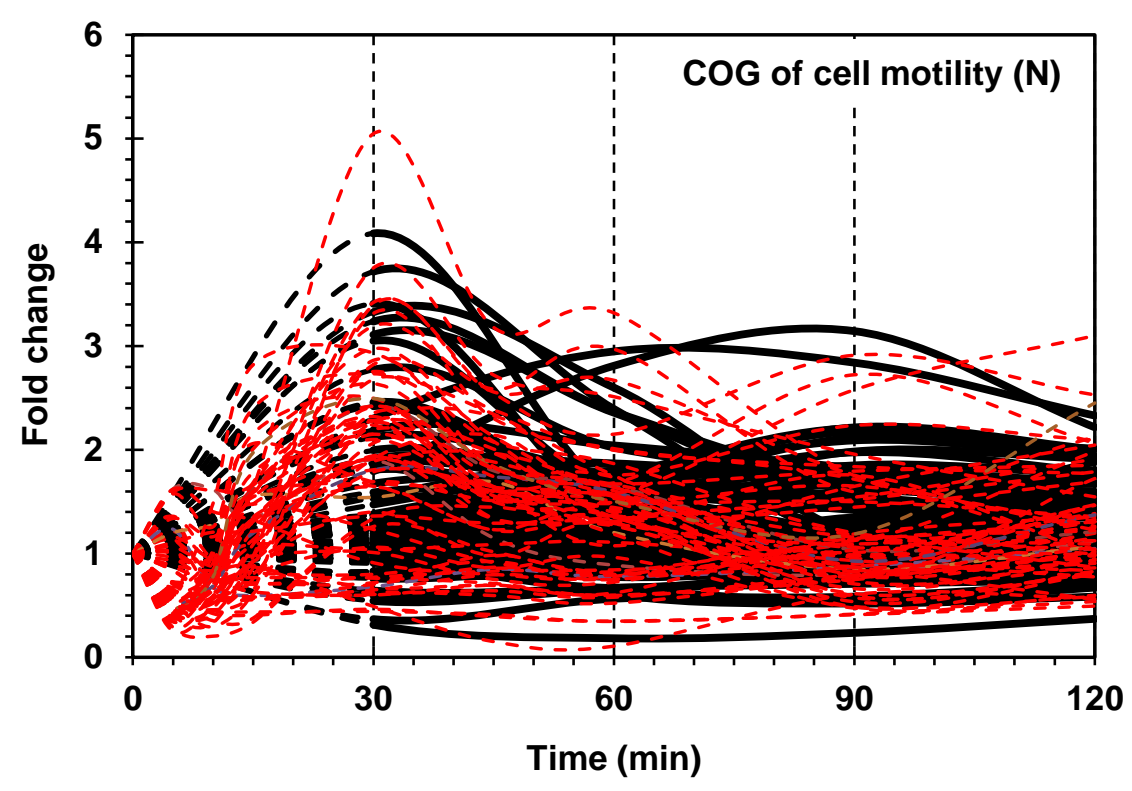

B

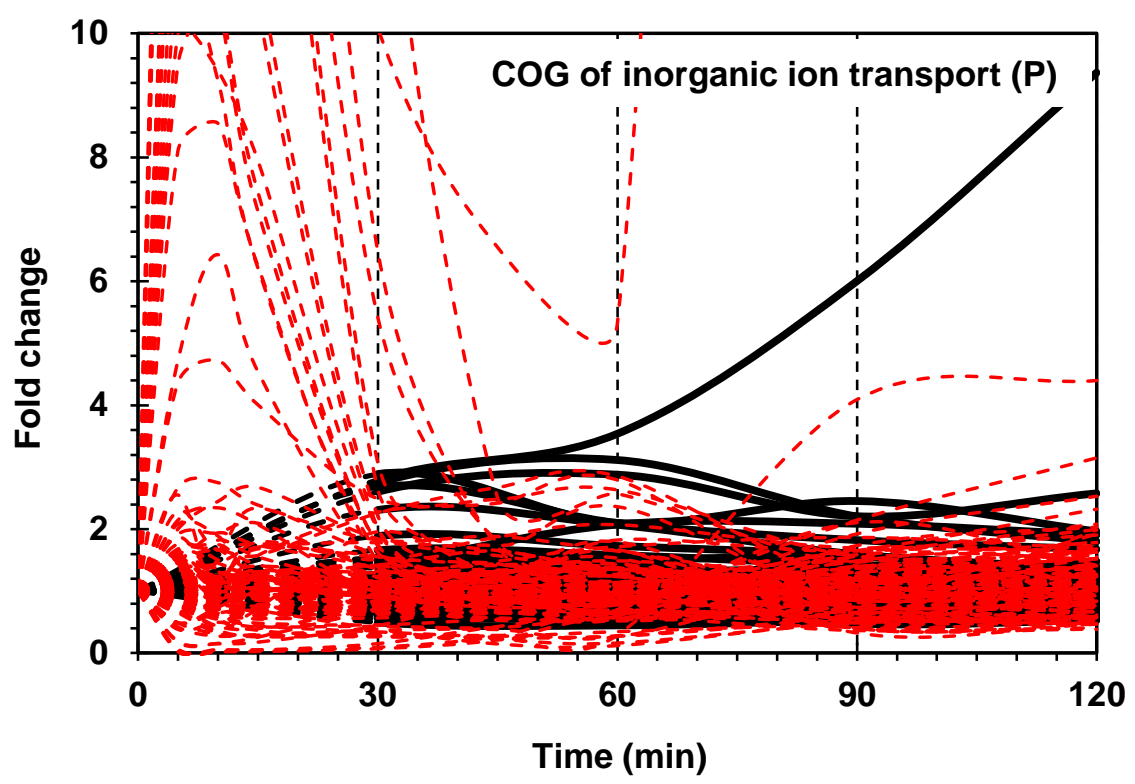




$\underbrace{}_{2,719,202}$ Group I-A

B
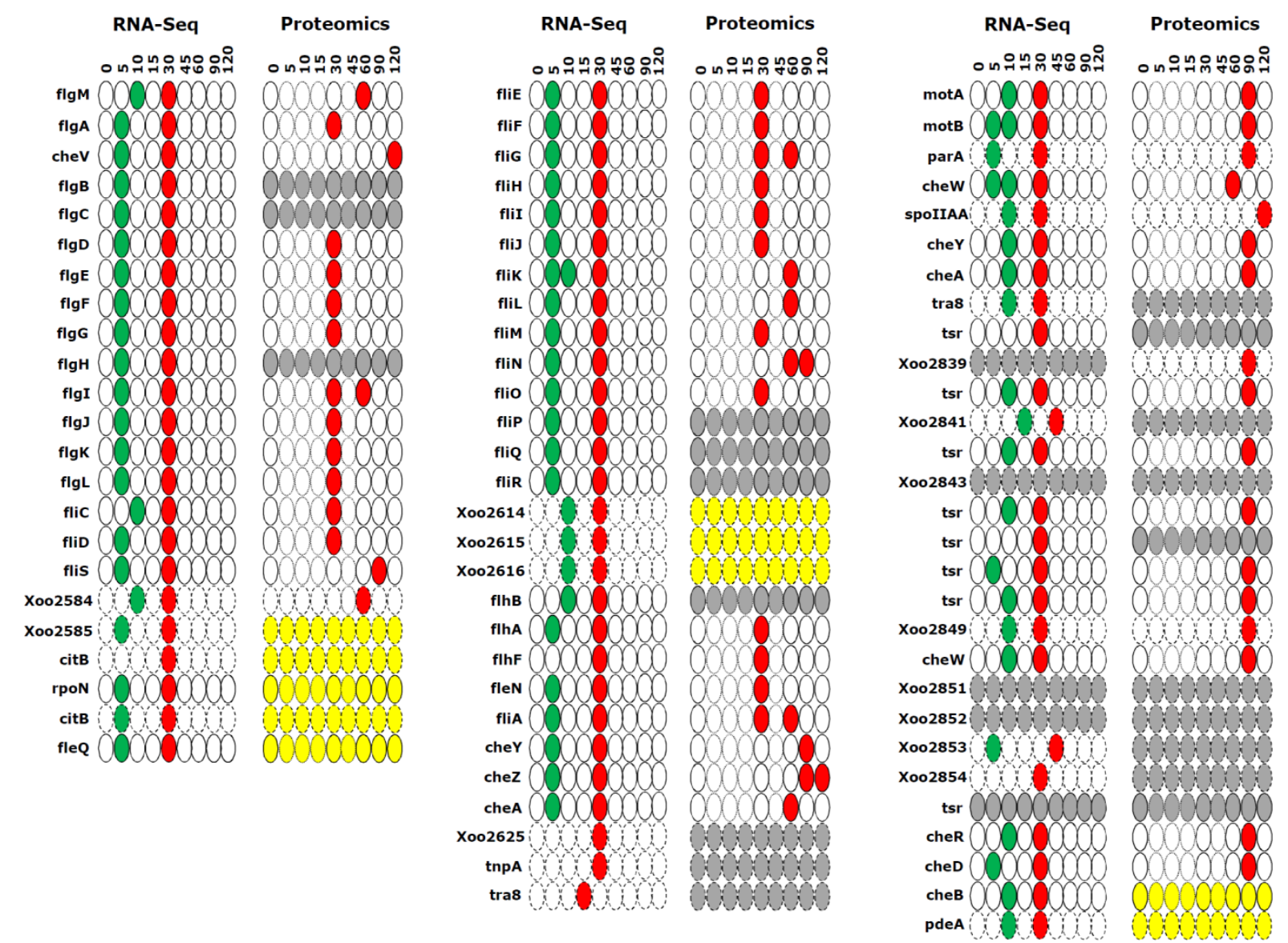
A
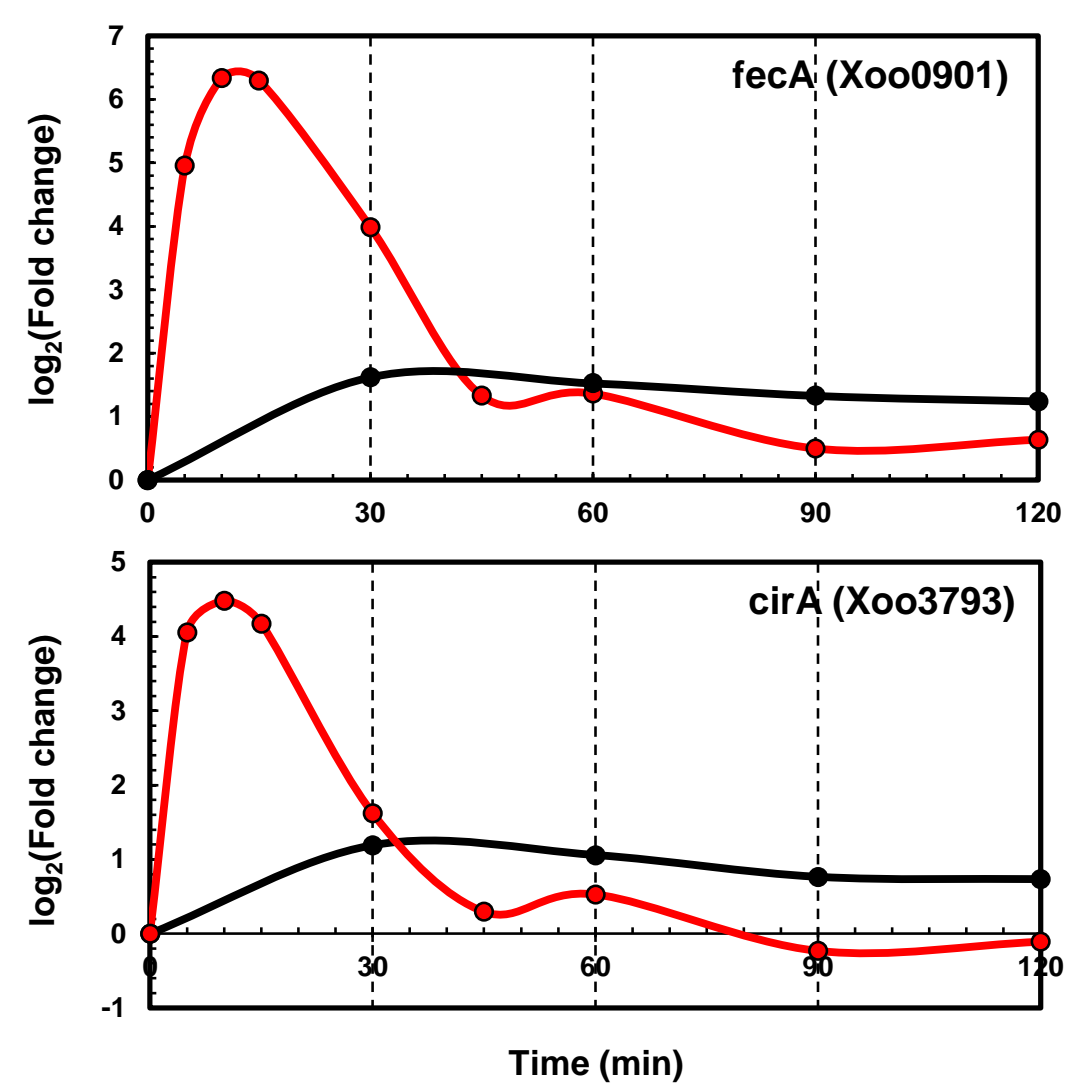

B
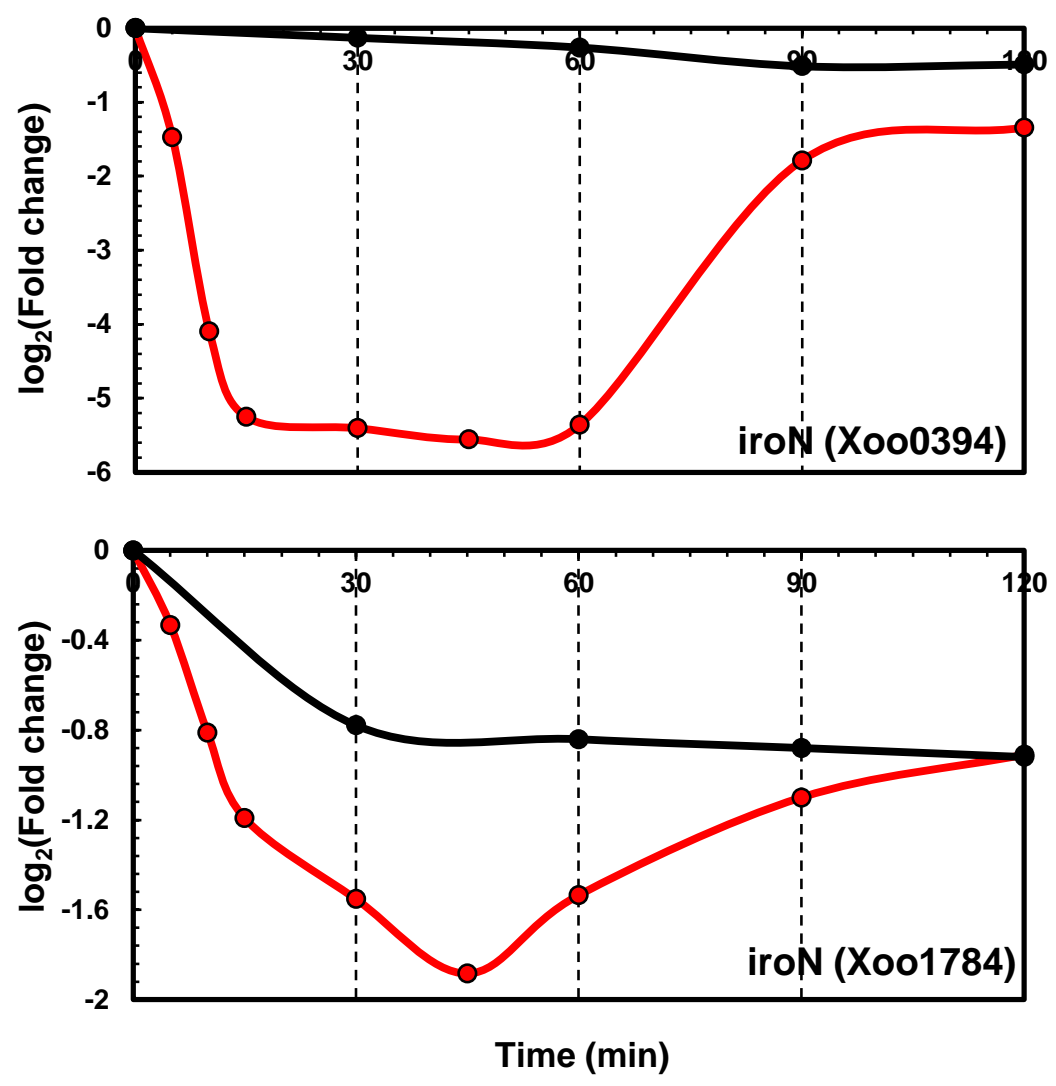
RLX (unknown pathogenic signals from initial interaction)

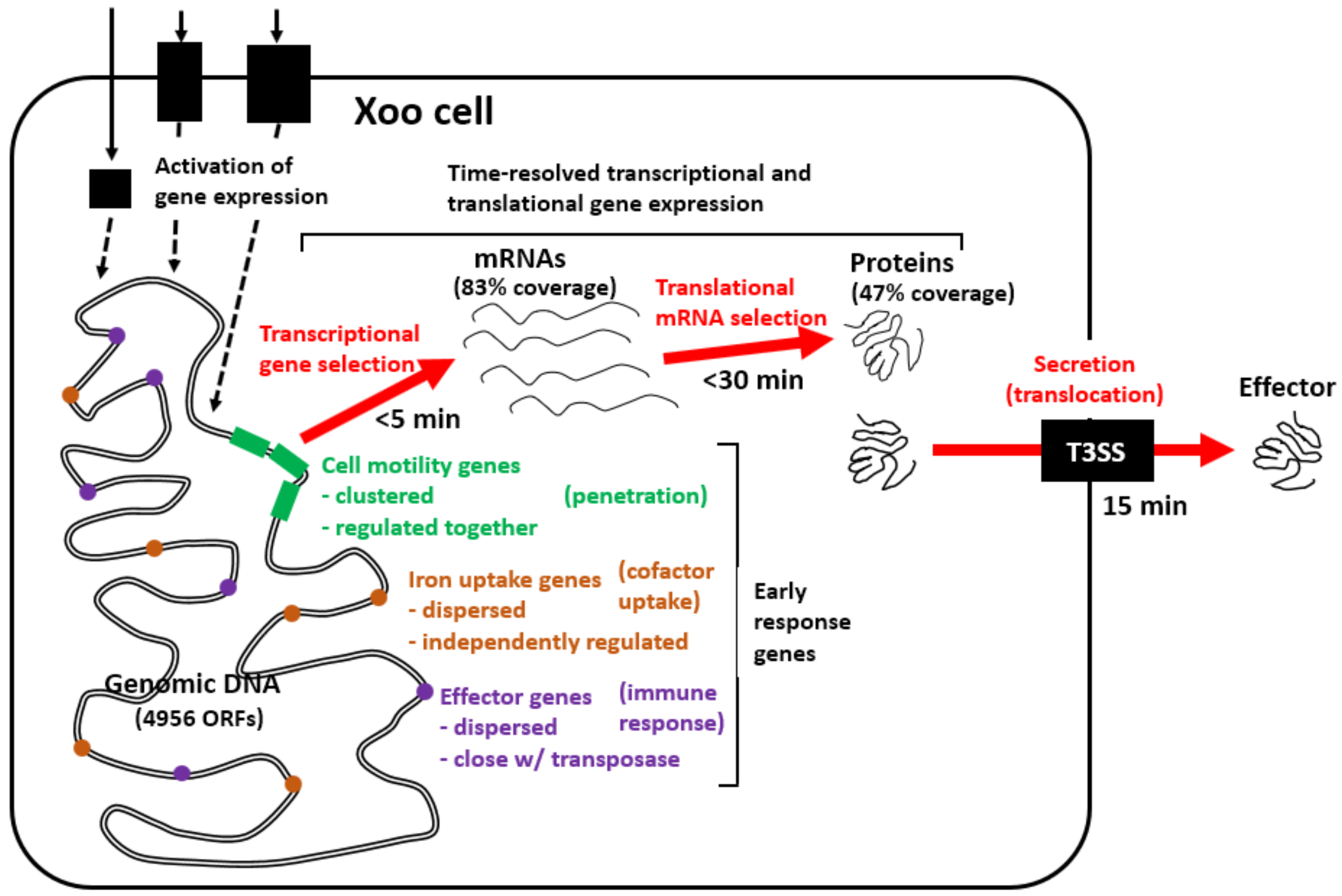

